\title{
Review
}

\section{Lupins and Health Outcomes: A Systematic Literature Review}

\author{
Lesley Bryant ${ }^{1}$, Anna Rangan ${ }^{1}\left[\right.$ and Sara Grafenauer ${ }^{2,3, *}$ \\ 1 Nutrition and Dietetics Group, Sydney Nursing School, Faculty of Medicine and Health, Charles Perkins \\ Centre, The University of Sydney, Camperdown, NSW 2006, Australia; lbry9477@uni.sydney.edu.au (L.B.); \\ anna.rangan@sydney.edu.au (A.R.) \\ 2 Grains \& Legumes Nutrition Council, Mount Street, Sydney, NSW 2060, Australia \\ 3 School of Medicine and Health, University of New South Wales, Randwick, NSW 2052, Australia \\ * Correspondence: s.grafenauer@unsw.edu.au
}

Citation: Bryant, L.; Rangan, A.; Grafenauer, S. Lupins and Health Outcomes: A Systematic Literature Review. Nutrients 2022, 14, 327. https://doi.org/10.3390/nu14020327 Academic Editors: Stuart Johnson and Rewati Bhattarai

Received: 17 December 2021

Accepted: 11 January 2022

Published: 13 January 2022

Publisher's Note: MDPI stays neutral with regard to jurisdictional claims in published maps and institutional affiliations.

Copyright: (C) 2022 by the authors. Licensee MDPI, Basel, Switzerland. This article is an open access article distributed under the terms and conditions of the Creative Commons Attribution (CC BY) license (https:// creativecommons.org/licenses/by/ $4.0 /)$.

\begin{abstract}
Lupins have a unique nutrient profile among legumes and may have beneficial health effects when included in the diet. The aim of this systematic review was to investigate the effects of lupin on a range of health outcome measures. Databases included MEDLINE, Embase and CINAHL, and focused on controlled intervention studies on healthy adults and those with chronic disease such as type 2 diabetes, cardiovascular disease and overweight. The Preferred Reporting Items for Systematic Reviews and Meta-Analyses protocol was followed. Investigated intervention diets utilised whole lupin, lupin protein or lupin fibre, and outcomes were measured by markers of chronic disease, body weight and satiety. Quality assessment of results was performed using the Cochrane revised risk of bias tool. Overall, 21 studies with 998 participants were included: 12 using whole lupin, four used lupin protein and five lupin fibre. Beneficial changes were observed in $71 \%$ of studies that measured blood pressure, $83 \%$ measuring satiety and $64 \%$ measuring serum lipids. Unintended weight loss occurred in $25 \%$ of studies. Whole lupin demonstrated more consistent beneficial effects for satiety, glycaemic control and blood pressure than lupin protein or lupin fibre. Heterogeneity, low study numbers and a small participant base indicated further studies are required to strengthen current evidence particularly regarding the protein and dietary fibre components of lupin.
\end{abstract}

Keywords: lupin; health outcomes; type 2 diabetes; cardiovascular disease; obesity

\section{Introduction}

Lupin (Lupinus) is a legume of the Fabaceae family that has formed part of the human diet since early civilisations. Legumes such as chickpeas, lentils, peas, beans and pulses continue to be a staple food in many parts of the world. Prized by consumers for their highly nutritious and inexpensive nature, they are becoming increasingly valued by producers for their ecological sustainability. With an increasing awareness of the benefits of regular legume consumption to human health, particularly among people living with chronic disease, lupin may be a useful addition to the food supply. Australia accounts for approximately $85 \%$ of the world's lupin production. It is grown predominantly in Western Australia, with some parts of New South Wales, Victoria and South Australia also under cultivation [1,2]. Lupin grows well in poor agricultural conditions, is pest-resistant and requires less water than many other food crops, therefore is ideally suited to Western Australia's climate and sandy soils. Moreover, it helps to improve soil fertility by the nitrogen fixing action of its rhizome, a characteristic shared with all legume plants [3]. The two most common varieties grown are the narrow-leafed lupin, also known as Australian sweet lupin or blue lupin (Lupinus angustifolius) and the white or albus lupin (Lupinus albus). Species of lupin cultivated globally include Lupinus mutabilis and Lupinus luteus or yellow lupin.

Macronutrient profiles of the various lupin species differ slightly. Compared with other legumes, Australian sweet lupin has one of the highest combinations of both protein 
and fibre. While protein can make up as much as $40 \%$ and fibre $30 \%$ as dry weight, with an additional 5\% inulin, its carbohydrate content accounts for less than $10 \%$ [4]. As with other legumes, lupins have a high nutrient density, yet their energy density is low. They are rich in minerals such as iron, magnesium, zinc, calcium and potassium; they contain vitamin A, B vitamins and vitamin E; and the fat profile predominantly consists of mono- and polyunsaturated fats, omega 3, 6 and 9 fatty acids [5]. Despite these benefits, Australians include very little lupin or other legumes in their diet, compared with populations in other countries. According to the Grains and Legumes Nutrition Council's 2017 consumption study [5], only $28 \%$ of people in Australia eat legumes, a modest $4 \%$ increase in three years. Conversely, secondary analysis of the National Nutrition and Physical Activity Survey (NNPAS 2011-12) [6] suggests consumption was around $4 \mathrm{~g}$ per day, perhaps indicating use as a snack rather than as a staple food within meals. According to dietary modelling, Australians will need to eat almost five times more legumes to meet the Australian Dietary Guidelines recommendation [7]. Lupin is an excellent source of high-quality plant protein for people who follow a vegetarian or vegan diet. It is gluten-free and provides a more nutrient-dense wheat replacement than other grain and cereal alternatives currently utilised in gluten-free diets. The Australian food industry is beginning to recognise the value of lupin and a range of lupin products is now available, including whole lupin flakes, flour, crumb, meal, kibble and splits. The addition of lupin into other foods enhances their nutritional value and may be an acceptable approach to introduce lupin into the food supply, particularly as an ingredient [8]. Flour made from whole lupin can be easily incorporated into many foods and isolated protein and fibre from the lupin seed may also be of benefit, though separation of the component parts is a more complex process. Interest in lupin as a functional food is increasing among food manufacturers, however it is not clear whether there is a difference in biological effect between whole lupin, lupin protein and lupin fibre when consumed as part of the diet. Sensitivity to legume protein allergens may have an inhibitory effect on lupin consumption in some individuals. In 2017 Food Standards Australia New Zealand included lupin among allergens that must be declared on food products, however, allergic response to lupin is milder and occurs less frequently compared with exposure to peanut and soy [9]. According to Allergy and Anaphylaxis Australia, it is estimated that less than 1\% of the Australian population is allergic to lupin [10].

Despite the increasing popularity of plant-based diets, it has been some years since a review of the health benefits of lupin intake was conducted. A 2015 review of the literature on lupins, among a broad range of other legumes, suggested Lupinus angustifolius may beneficially effect blood pressure, blood lipids, insulin sensitivity and the gut microbiome [8]. A 2016 review of the nutritional, chemical and health-promoting properties of Lupinus albus recognised its potential in the production of functional food [11]. A systematic literature review in 2020 on the effects of legume consumption on markers of glycaemic control in people with and without diabetes excluded studies of less than six weeks' duration, therefore post-meal and short-duration lupin interventions were not captured [12]. The aim of this systematic review was to investigate the effects of lupin on a range of health outcome measures.

\section{Materials and Methods}

This systematic literature review was conducted in accordance with the Preferred Reporting Items for Systematic Reviews and Meta-analyses (PRISMA) guidelines [13]. The study protocol was submitted to the International Prospective Register of Systematic Reviews (PROSPERO) for registration (TBC).

\subsection{Eligibility and Exclusion Criteria}

To research the question 'Is there an effect of lupin consumption on health outcomes in humans?' a framework was developed using the PICO (Population, Intervention, Comparator/Control, Outcome) format (Table S1). Studies were eligible for inclusion in 
this review if they met the following criteria: (a) controlled intervention trial of any duration and of parallel or cross-over design; (b) populations comprising any adults aged 18 years and above, with or without chronic disease, overweight or obesity; (c) dietary interventions in the form of whole lupin, such as dried, pickled and brined seeds, flakes, flour, crumb, meal, kibble and splits, or components of lupin such as protein and fibre; (d) assessed the effect of lupin consumption on biomarkers of chronic disease such as any related to diabetes, cardiovascular disease, hypertension, hypercholesteremia, hyperlipidaemia, cancer, inflammation, and oxidative stress, or assessed the effect of lupin consumption on anthropometric measurements and perceptions of satiety in relation to overweight and obesity and their association with chronic disease risk. The exclusion criteria applied to the study search were: (a) participants below 18 years of age; (b) assessments of single isolated proteins, peptides or alkaloids from lupin; (c) lupin intake as a supplement in capsule form; (d) non-English language studies; (e) publication dates before 1 January 2000.

\subsection{Search Strategy}

The following databases were searched: EMBASE (via Ovid) MEDLINE (via Ovid), CINHAHL (via EBSCO) from 1 January 2000 until 13 September 2021. Reference lists of eligible studies were scanned and searched manually on PubMed for additional studies.

\subsection{Study Selection, Data Extraction, and Quality Assessment}

Search results were imported into EndNote $X 9^{\circledR}$ referencing software (EndNote X9, Clarivate Analytics, Philadelphia, PA, USA) and duplicates were removed. Screening of studies was performed in two stages, first by title and abstract, then by full text. A data extraction form was created in Microsoft ${ }^{\circledR}$ Excel $^{\circledR}$ spreadsheet (Microsoft 365 MSO Version 2109.14430.20306, Redmond, WA, USA) to include study citation, design, and duration; participant numbers and characteristics; intervention and control diet information; outcomes measured, and results obtained. The included studies were assessed for withinstudy risk of bias using the revised Cochrane risk-of-bias tool (RoB2) for randomised controlled trials [14]. Reviewer L.B. assessed studies to determine whether each study had low, some concerns, or high risk of bias. Areas of uncertainty were resolved in consultation with a second reviewer (S.G.). Assessment domains included risk of bias arising from the randomisation process, period and carryover effects, deviations from intended interventions, missing outcome data, measurement of the outcome, and selection of the reported result [14].

\section{Results}

\subsection{Search Results and Study Selection}

The search was conducted on 13 September 2021, returning a total of 157 records. One additional paper was identified from the reference lists of eligible studies. Following automated removal of duplicates by Endnote X9, 127 studies remained. Screening by title and abstract excluded 94 studies. Principal reasons for exclusion were publication type and study aim, interventions and measured outcomes beyond the scope of this review. A full-text review of the remaining 33 led to the exclusion of a further 12 studies. Reasons for exclusion were: duplications in other journals and/or non-English languages $(n=4)$, brief conference or workshop communications $(n=2)$, lupin intervention administration in capsule or supplement form $(n=2)$, investigation of lupin fractions such as alkaloids or single proteins $(n=3)$ and in vitro study protocol $(n=1)$. A total of 21 journal articles of controlled intervention studies met the inclusion criteria and were included in this qualitative review (Figure 1). Two studies generated three articles that reported on different sets of variables within each. These articles were treated as stand-alone studies and included in the final total. 


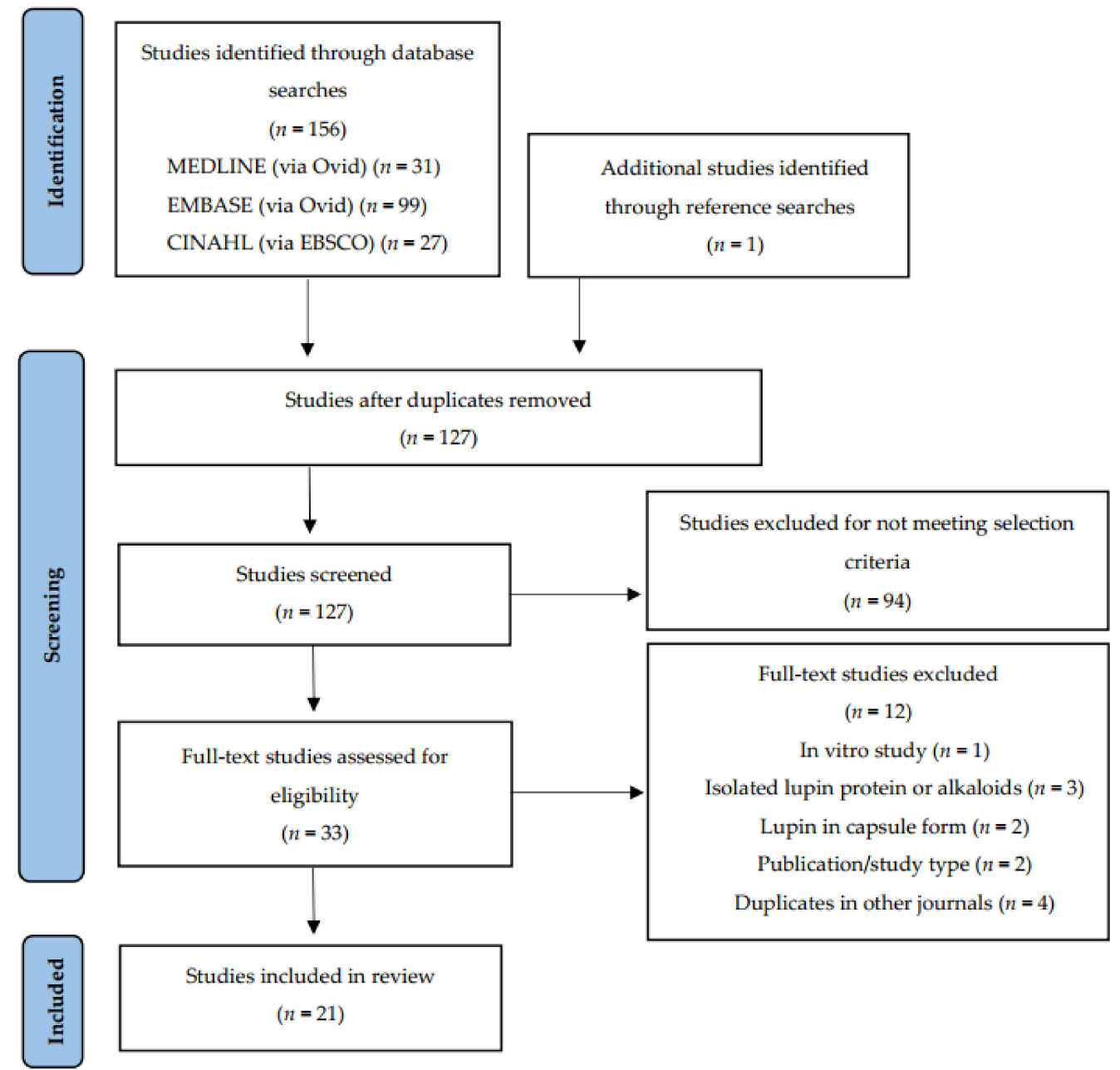

Figure 1. Preferred Reporting Items for Systematic Reviews and Meta-Analyses (PRISMA) flow diagram for study selection.

\subsection{Study Characteristics}

Six of the included studies were randomised controlled trials (RCTs) of parallel design, the remaining 15 were cross-over studies, of which 11 were RCTs, while two were controlled, non-randomised, crossover studies. Research was based predominantly in Australia with smaller number of studies conducted in Germany, Italy and Ecuador. Table 1 lists study locations and the species of lupin under investigation. Five studies recruited healthy men and women [15-19], three recruited healthy men only [20-22], four studies involved men and women with type 2 diabetes [23-26], five with hypercholesterolaemia [27-31] and four involved people who were overweight or obese [32-35]. The number of completing participants ranged from $n=5$ to $n=175$ per study. Approximately $25 \%$ of the 998 participants across all studies were classified as healthy. Duration of study periods ranged from post-meal studies to 12 months. Categorisation of studies by form of lupin administered during treatment phases identified three distinct groups: whole lupin, lupin protein and lupin fibre. Study characteristics and outcomes of 12 whole lupin studies are summarised in Table 2, four lupin protein studies in Table 3, and five lupin fibre studies in Table 4. Measured outcomes were multiple and varied across studies, with $p<0.05$ being declared as statistically significant. 
Table 1. Study location, lupin species and form of lupin consumed in eligible studies investigating the health benefits of lupin consumption.

\begin{tabular}{|c|c|c|c|}
\hline Country & Reference & $\begin{array}{l}\text { Lupin Species/Common Name } \\
\text { (NS = Not Stated) }\end{array}$ & $\begin{array}{c}\text { Whole Seed (W), Protein Isolate (PI) } \\
\text { or Fibre Isolate (FI) }\end{array}$ \\
\hline \multirow[t]{13}{*}{ Australia } & Hall et al., 2005 [18] & L. angustifolius & $\mathrm{W}$ \\
\hline & Hall et al., 2005 [22] & L. angustifolius & FI \\
\hline & Smith et al., 2006 [20] & L. angustifolius & FI \\
\hline & Johnson et al., 2006 [21] & L. angustifolius & FI \\
\hline & Lee et al., 2006 [16] & NS & W \\
\hline & Lee et al., 2009 [33] & NS & W \\
\hline & Yang et al., 2010 [32] & NS & W \\
\hline & Hodgson et al., 2010 [34] & L. angustifolius & W \\
\hline & Dove et al., 2011 [26] & L. angustifolius & W \\
\hline & Keogh et al., 2011 [17] & NS & W \\
\hline & Belski et al., 2011 [35] & L. angustifolius & W \\
\hline & Skalkos et al., 2020 [24] & Australian Sweet Lupin & W \\
\hline & Ward et al., 2020 [23] & L. angustifolius & W \\
\hline \multirow[t]{6}{*}{ Germany } & Weiße et al., 2010 [27] & L. angustifolius & PI \\
\hline & Bähr et al., 2013 [30] & L. angustifolius & PI \\
\hline & Fechner et al., 2013 [19] & L. angustifolius and L. albus & FI \\
\hline & Fechner et al., 2014 [29] & L. angustifolius & FI \\
\hline & Bähr et al., 2015 [31] & L. angustifolius & PI \\
\hline & Schopen et al., 2017 [15] & L.albus & W \\
\hline Italy & Sirtori et al., 2012 [28] & L. angustifolius & PI \\
\hline Ecuador & Fornasini et al., 2019 [25] & L. mutabilis & W \\
\hline
\end{tabular}


Table 2. Characteristics and major outcomes of studies examining whole lupin consumption and health outcomes.

\begin{tabular}{|c|c|c|c|c|c|c|c|}
\hline Reference & Study Type & $\begin{array}{l}\text { Subjects }(n) \text { and } \\
\text { Characteristics }\end{array}$ & Intervention & Control/Comparator & Energy Balance & Main Health Markers & Main Outcomes \\
\hline $\begin{array}{l}\text { Hall et al., } \\
2005 \text { [18] }\end{array}$ & $\begin{array}{l}\text { RCT single blind } \\
\text { cross-over Post-meal } \\
\text { study }\end{array}$ & $\begin{array}{c}n=11 \\
\text { Healthy men }(n=9) \text { and } \\
\text { women }(n=2) \text {. Mean age } \\
31.6 \text { years, } \\
\text { range } 25-45 \text { years }\end{array}$ & $\begin{array}{l}\text { Breakfast including lupin } \\
\text { bread with } 10 \% \text { wheat } \\
\text { flour replaced with } \\
\text { Australian sweet lupin } \\
\text { kernel flour }\end{array}$ & $\begin{array}{l}\text { Breakfast including } \\
\text { standard recipe white } \\
\text { bread }\end{array}$ & $\begin{array}{l}\text { 95kJ difference in lupin } \\
\text { breakfast }(1338 \mathrm{~kJ}) \text { and } \\
\text { control breakfast }(1243 \mathrm{~kJ})\end{array}$ & $\begin{array}{c}120 \text { min SG, SI } \\
180 \text { min satiety response. } \\
\text { Glycaemic index (GI), } \\
\text { insulinaemic index (II) and } \\
\text { satiety index (WB }=100) \text {. } \\
\text { Energy intake from ad } \\
\text { libitum buffet and during } \\
\text { remainder of day. }\end{array}$ & $\begin{array}{c}\downarrow \mathrm{GI}(p=0.022) \\
\uparrow \mathrm{II}(p=0.046) \\
\text { Trend to lower SG at } 30 \mathrm{~min} . \\
\text { Peak satiety at } 10 \mathrm{~min} \text { WB and } 25 \mathrm{~min} \text { lupin. } \\
\text { Below baseline at } 160 \mathrm{~min} \text { for WB. Lupin did } \\
\text { not reach zero within } 180 \text { min. } \\
\text { No difference in SI, satiety response, satiety } \\
\text { index, energy intake at ad libitum buffet, or } \\
\text { energy intake during remainder of day. }\end{array}$ \\
\hline \multirow[t]{2}{*}{$\begin{array}{l}\text { Lee et al., } \\
2006 \text { [16] }\end{array}$} & $\begin{array}{c}\text { RCT cross-over Study } \\
\text { 1: } 4 \text { treatments } 1 \text { week } \\
\text { apart }\end{array}$ & $\begin{array}{c}\text { Study } 1: n=16 \\
\text { healthy men }(n=8) \text { and } \\
\text { women }(n=8) \text {. } \\
\text { Mean age } 58.6 \pm 7.2 \text { years. } \\
\text { Mean BMI } \\
31.3 \pm 4.5 \mathrm{~kg} / \mathrm{m}^{2}\end{array}$ & $\begin{array}{l}\text { Lupin bread } 40 \% \text { total } \\
\text { flour ( } 24 \% \text { final weight of } \\
\text { bread) in } 4 \text { treatments: } \\
\text { WB-WB/WB-lupin/lupin- } \\
\text { WB/lupin-lupin }\end{array}$ & $\begin{array}{l}\text { White bread breakfast and } \\
\text { lunch }\end{array}$ & $\begin{array}{l}\text { Isocaloric at breakfast, } \\
\text { ad libitum lunch }\end{array}$ & $\begin{array}{l}\text { Post breakfast } 180 \mathrm{~min} \\
\text { satiety response. Total } \\
\text { energy intake after } a d \\
\text { libitum lunch. }\end{array}$ & $\begin{array}{c}\uparrow \text { Satiety at breakfast for satisfaction and } \\
\text { prospective consumption }(p<0.001, p<0.001) \text {. } \\
\uparrow \text { Satiety at } 180 \text { min for fullness }(p<0.001), \\
\text { satisfaction }(p<0.001) \text { and prospective } \\
\text { consumption }(p<0.001) \text {. } \\
\downarrow \text { Energy intake at lunch after lupin breakfast } \\
(-488 ; 95 \% \text { CI: }-798,-178 \mathrm{~kJ}) \text {. } \\
\downarrow \text { Intrameal energy intake when lupin } \\
\text { consumed at lunch }(-1028 ; 95 \% \text { CI: }-1338, \\
-727 \mathrm{~kJ}) .\end{array}$ \\
\hline & $\begin{array}{l}\text { Study 2: } 2 \text { treatments } \\
1 \text { week apart }\end{array}$ & $\begin{array}{c}\text { Study } 2: n=17 \\
\text { healthy men }(n=11) \text { and } \\
\text { women }(n=6) \text {. } \\
\text { Mean age } 61.0 \pm 5.6 \text { years. } \\
\text { Mean BMI } \\
27.2 \pm 4.3 \mathrm{~kg} / \mathrm{m}^{2}\end{array}$ & $\begin{array}{l}\text { Lupin bread } 40 \% \text { wheat } \\
\text { flour replaced with lupin } \\
\text { kernel flour }\end{array}$ & White bread & Isocaloric & $\begin{array}{l}\text { Post breakfast } 180 \mathrm{~min} \\
\text { plasma ghrelin, SG and SI }\end{array}$ & $\begin{array}{c}\text { Altered ghrelin response }(p=0.04) \\
\downarrow 180 \text { min plasma ghrelin }(p=0.009) \\
\text { Altered glucose response }(p=0.01) \\
\quad \downarrow \text { glucose AUC }(p=0.006) \\
\downarrow \text { insulin AUC }(p=0.002) .\end{array}$ \\
\hline $\begin{array}{l}\text { Lee et al., } \\
2009 *[33]\end{array}$ & $\begin{array}{l}\text { RCT parallel study } \\
16 \text { weeks } 2 \text { cohorts }\end{array}$ & $\begin{array}{c}n=74 \\
\text { Overweight and obese } \\
\text { men }(n=26) \text { and women } \\
(n=48) \\
\text { Mean age } 59.0 \pm 7.4 / \\
56.8 \pm 8.5 \text { years. } \\
\text { Mean BMI } 30.6 \pm 3.6 / \\
30.5 \pm 3.4 \mathrm{~kg} / \mathrm{m}^{2} .\end{array}$ & $\begin{array}{l}\text { Lupin bread } 40 \% \text { wheat } \\
\text { flour replaced with lupin } \\
\text { kernel flour (24\% final } \\
\text { weight bread) to replace } \\
\text { usual carbohydrate-rich } \\
\text { foods to } 15-20 \% \text { usual } \\
\text { energy intake. }\end{array}$ & $\begin{array}{l}\text { White bread to replace } \\
\text { normal bread intake and } \\
\text { other carbo-hydrate-rich } \\
\text { foods to } \sim 15-20 \% \text { usual } \\
\text { energy intake }\end{array}$ & Isocaloric & $\begin{array}{l}\text { 24-h SBP, DBP, pulse } \\
\text { pressure and heart rate }\end{array}$ & $\begin{array}{c}\downarrow 24-\mathrm{h} \text { SBP }(p=0.03) \\
\downarrow \text { pulse pressure }(p<0.001) \text {. } \\
\text { No difference in DBP, heart rate }\end{array}$ \\
\hline $\begin{array}{l}\text { Yang et al., } \\
2010^{*}[32]\end{array}$ & $\begin{array}{l}\text { Paper refers to the Lee } \\
\text { et al., } 2009 \text { study } \\
\text { above }\end{array}$ & As above & As above & As above & As above & $\begin{array}{c}\text { Plasma and urinary } \\
\text { F2-isoprostanes, plasma } \\
\text { 20-HETE, plasma and } \\
\text { urinary nitrite and nitrate } \\
\text { concentrates }\end{array}$ & No difference between groups \\
\hline
\end{tabular}


Table 2. Cont.

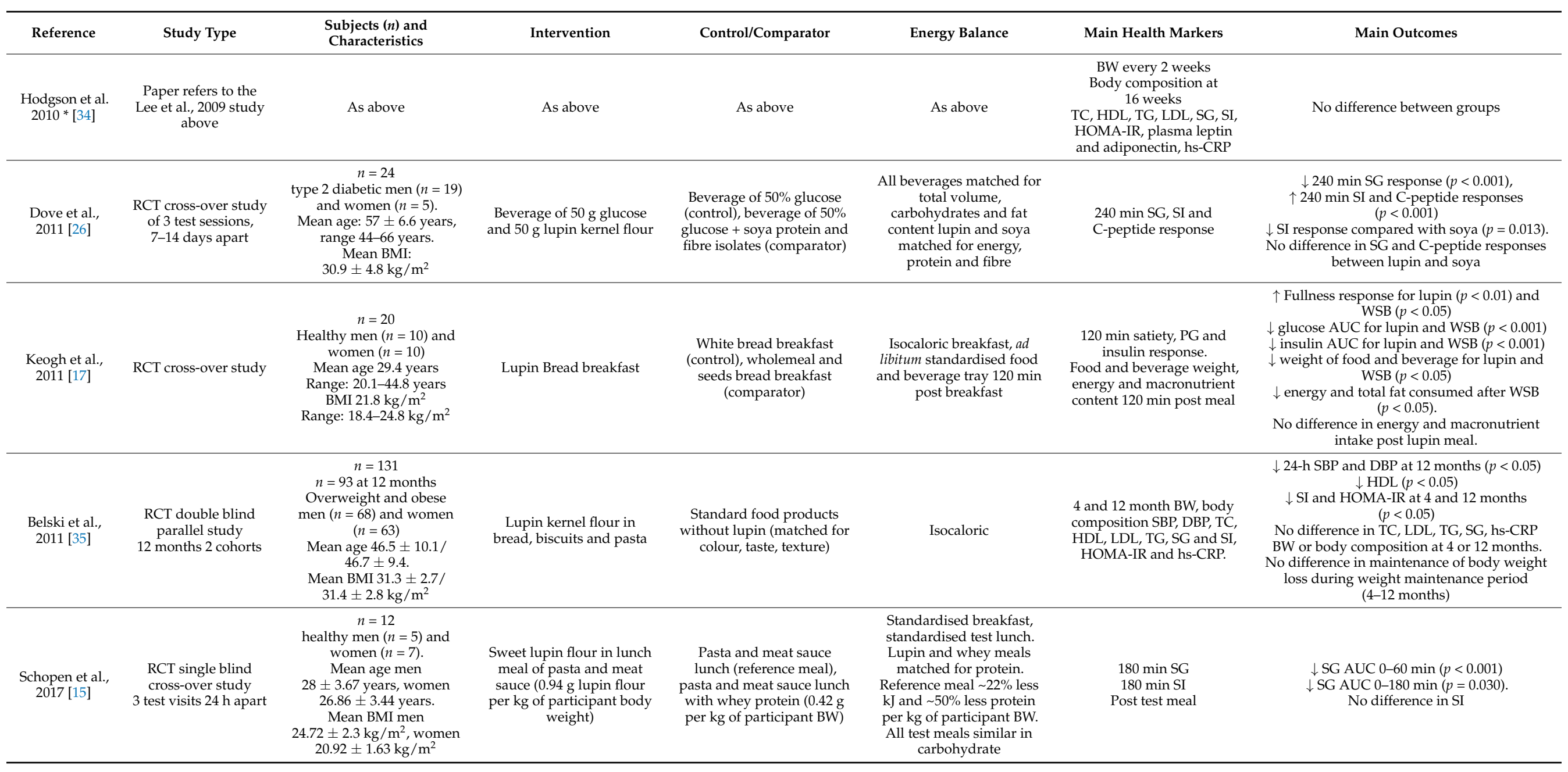


Table 2. Cont.

\begin{tabular}{|c|c|c|c|c|c|c|c|}
\hline Reference & Study Type & $\begin{array}{l}\text { Subjects }(n) \text { and } \\
\text { Characteristics }\end{array}$ & Intervention & Control/Comparator & Energy Balance & Main Health Markers & Main Outcomes \\
\hline $\begin{array}{c}\text { Fornasini } \\
\text { et al., 2019 } \\
\text { [25] }\end{array}$ & $\begin{array}{l}\text { Controlled } \\
\text { non-randomised } \\
\text { single blind } \\
\text { cross-over study } \\
\text { (28 weeks) } \\
\text { (One-group } \\
\text { pretest-posttest } \\
\text { design with double } \\
\text { pretest) }\end{array}$ & $\begin{array}{c}n=5 \\
\text { type } 2 \text { diabetic men }(n=19) \\
\text { and women }(n=32) \text { under } \\
\text { conventional non-insulin } \\
\text { medication. } \\
\text { Mean age } 64.1 \pm 11.1 \text { years. } \\
\text { Mean BMI } \\
30.3 \pm 4.5 \mathrm{~kg} / \mathrm{m}^{2}\end{array}$ & $\begin{array}{l}\text { Whole Lupinus mutabilis } \\
10 \mathrm{~g} \text { dehydrated lupin } \\
\text { snack. One dose per day } \\
7 \text { weeks (following initial } \\
\text { 14-week medication only } \\
\text { period). } 2 \text { doses per day } \\
\text { next } 7 \text { weeks }\end{array}$ & Usual diet and medication & $\mathrm{N} / \mathrm{A}$ & $\begin{array}{l}\text { At } 14 \text { and } 28 \text { weeks } \\
\text { BW, SBP, DBP, SG, SI, } \\
\text { HbA1c, TC, LDL, HDL, } \\
\text { Uric acid, CRP }\end{array}$ & $\begin{array}{c}\uparrow \text { SG and SI } 0-28 \text { weeks }(p \leq 0.05) \\
\uparrow \text { HDL } 0-28 \text { weeks }(p \leq 0.05) \\
\downarrow \text { BW }(p=0.015) \text { and BMI }(p=0.009) \\
0-28 \text { weeks } \\
\downarrow \text { SBP and DBP } 0-28 \text { and } 14-28 \text { weeks } \\
(p \leq 0.05) . \\
\text { No difference in HbA1c, TC, LDL, uric } \\
\text { acid, CRP }\end{array}$ \\
\hline $\begin{array}{l}\text { Skalkos et al., } \\
2020[24]\end{array}$ & $\begin{array}{c}\text { Controlled } \\
\text { non-randomised } \\
\text { cross-over study } \\
3 \text { consecutive days, } \\
1 \text { treatment per day }\end{array}$ & $\begin{array}{c}n=20 \\
\text { Post-surgical hospital } \\
\text { patient men }(n=12) \text { and } \\
\text { women }(n=8) \text { with type } \\
2 \text { diabetes. } \\
\text { Mean age } 74.3 \pm 11.7 \text { years. } \\
\text { Mean BMI } \\
30.7 \pm 4.5 \mathrm{~kg} / \mathrm{m}^{2}\end{array}$ & $\begin{array}{l}4 \times \text { lupin biscuit } \\
\text { containing } 20 \% \text { lupin flour } \\
\text { ( } 2 \text { at morning tea and } 2 \text { at } \\
\text { afternoon tea) on day } 1\end{array}$ & $\begin{array}{c}4 \times \text { wholemeal spelt } \\
\text { biscuit day } 2 \\
4 \times \text { Arnott's Marie biscuit } \\
\text { (standard hospital option) } \\
\text { day } 3\end{array}$ & $\begin{array}{l}\text { Lupin and spelt biscuits } \\
\text { isocaloric }(1590 \mathrm{~kJ} / 100 \mathrm{~g}) \\
\text { and lower than Marie } \\
\text { biscuit }(1850 \mathrm{~kJ} / 100 \mathrm{~g}) \text {. } \\
\text { Higher protein, fat and } \\
\text { fibre in lupin and spelt, } \\
\text { and lower carbohydrate } \\
\text { and sugar than Marie } \\
\text { biscuit }\end{array}$ & $\begin{array}{l}\text { CGM interstitial glucose } \\
\text { pre- and } 5 \text { timepoints } \\
\text { post-meal, } \\
\text { bowel function (Bristol } \\
\text { Stool Chart), } \\
\text { hunger and fullness rating }\end{array}$ & $\begin{array}{l}\downarrow \text { glucose after dinner following lupin biscuit } \\
\qquad(p<0.001) \\
\text { No difference in } 0-90 \text { min glucose at } \\
\text { breakfast, morning tea, lunch, and afternoon } \\
\text { tea for all } 3 \text { treatments. } \\
\text { More patients felt fuller between afternoon } \\
\text { tea-dinner following lupin biscuit ( } p=0.018) \text {. } \\
\text { No difference in bowel function }\end{array}$ \\
\hline $\begin{array}{l}\text { Ward et al., } \\
2020[23]\end{array}$ & $\begin{array}{c}\text { RCT double blind } \\
\text { cross-over study } \\
\text { 1-week run-in period, } \\
2 \times 8 \text {-week treatment } \\
\text { with 8-week washout } \\
\text { period }\end{array}$ & $\begin{array}{c}n=22 \\
n=17 \text { completed } \\
\text { men }(n=14) \text { and women } \\
(n=8) \text { with } \\
\text { moderate-to-well } \\
\text { controlled type } 2 \text { diabetes } \\
(\text { HbAlc }<9 \%) \\
\text { Mean age } 58 \pm 6.6 \text { years. } \\
\text { Mean BMI } \\
29.9 \pm 3.5 \mathrm{~kg} / \mathrm{m}^{2}\end{array}$ & $\begin{array}{l}\text { Lupin-enriched foods } \\
\text { replacing } 20 \% \text { of daily } \\
\text { energy intake. Consumed } \\
\text { every breakfast, lunch and } \\
\text { at least } 3 \text { dinners per week. } \\
\text { Average daily intake } \sim 45 \mathrm{~g} \\
\text { lupin per day ( } 12 \mathrm{~g} / \mathrm{d} \\
\text { protein } 10 \mathrm{~g} / \mathrm{d} \text { fibre) }\end{array}$ & Wheat-based control foods & Isocaloric & $\begin{array}{l}\text { SG (at waking, } 1 \mathrm{~h} \text { post } \\
\text { breakfast, immediately } \\
\text { pre-lunch and } 1 \mathrm{~h} \\
\text { post-lunch), } \\
\text { SI, HOMA-IR, BW, BP, TC, } \\
\text { LDL, TG, HDL, C-peptide }\end{array}$ & $\begin{array}{l}\text { No difference between treatments. Borderline } \\
\text { significant decrease in TG with lupin }\end{array}$ \\
\hline
\end{tabular}

* Part of one study; Abbreviations: Area under the curve (AUC); Body mass index (BMI); Blood pressure (BP); Body weight (BW); Continuous glucose monitor (CGM); Diastolic blood pressure (DBP); Glycated haemoglobin (HbA1c); High density lipoprotein cholesterol (HDL); Homeostasis model assessment of insulin resistance (HOMA-IR); High-sensitivity C-reactive protein (hs-CRP); Low density lipoprotein cholesterol (LDL); Plasma glucose (PG); Systolic blood pressure (SBP); Serum glucose (SG); Serum insulin (SI); Triglycerides (TG); Total cholesterol (TC) 
Table 3. Characteristics and major outcomes of studies examining lupin protein consumption and health outcomes.

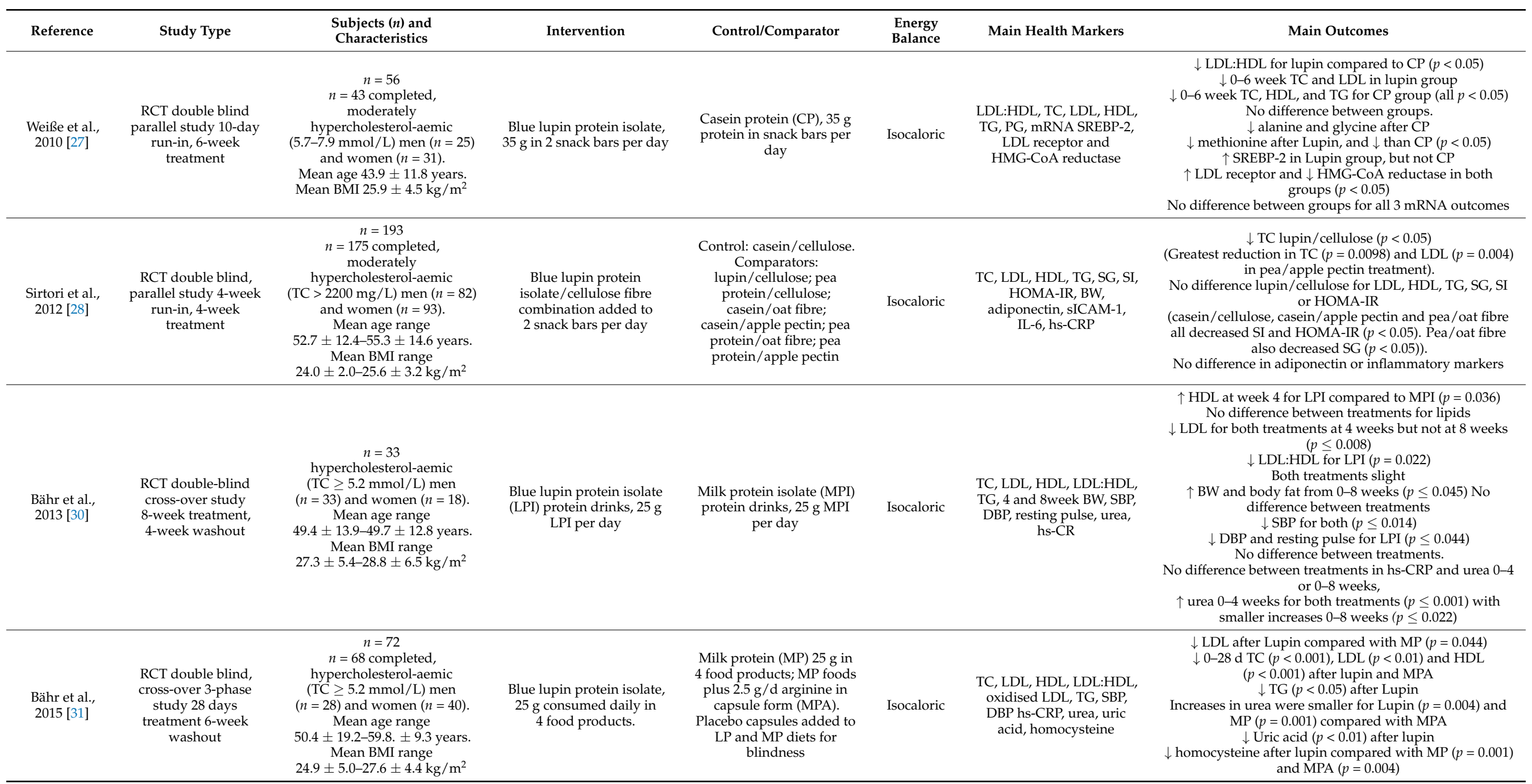

Abbreviations: Body mass index (BMI); Body weight (BW); Diastolic blood pressure (DBP)); High density lipoprotein cholesterol (HDL); Homeostasis model assessment of insulin resistance (HOMA-IR); High-sensitivity C-reactive protein (hs-CRP); Interleukin-6 (IL-6); Low density lipoprotein cholesterol (LDL); Plasma glucose (PG); Systolic blood pressure (SBP); Serum glucose (SG); Serum insulin (SI); soluble intracellular cell adhesion molecule-1 (sICAM-1); Triglycerides (TG); Total cholesterol (TC). 
Table 4. Characteristics and major outcomes of studies examining lupin fibre consumption and health outcomes.

\begin{tabular}{|c|c|c|c|c|c|c|c|}
\hline Reference & Study Type & Subject $(n)$ and Characteristics & Intervention & Control/Comparator & $\begin{array}{c}\text { Energy } \\
\text { Balance }\end{array}$ & Main Health Markers & Main Outcomes \\
\hline $\begin{array}{l}\text { Hall et al., } \\
2005 * \text { [22] }\end{array}$ & $\begin{array}{c}\text { RCT single blind } \\
\text { cross-over study } \\
28 \text { days of treatment } \\
28 \text { days washout period }\end{array}$ & $\begin{array}{c}n=44 \\
n=38 \text { completed, } \\
\text { healthy men. } \\
\text { Mean age } 41.0 \pm 1.9 \text { years. } \\
\text { Mean BMI } 26.7 \pm 0.5 \mathrm{~kg} / \mathrm{m}^{2}\end{array}$ & $\begin{array}{c}\text { Australian sweet lupin } \\
\text { kernel fibre in foods within } \\
\text { prescribed diet. } \\
55 \mathrm{~g} \text { dietary fibre/day for } \\
\text { diets }>9 \mathrm{MJ} / \text { day, }, 35 \mathrm{~g} \\
\text { dietary fibre /ay for diets } \\
\leq 9 \mathrm{MJ} / \text { day }\end{array}$ & $\begin{array}{c}\text { Prescribed control diet } \\
\text { without added lupin fibre. } \\
25 \mathrm{~g} \text { dietary fibre } / \text { day for } \\
\text { diets }>9 \mathrm{MJ} / \text { day, } 18 \mathrm{~g} \\
\text { dietary fibre/day for diets } \\
\leq 9 \mathrm{MJ} / \text { day }\end{array}$ & Isocaloric & $\begin{array}{c}\text { TC, HDL, TG, PG and } \\
\text { insulin, HOMA-IR, satiety } \\
\text { perception, BW }\end{array}$ & $\begin{array}{l}\downarrow \text { TC, LDL, TC:HDL and LDL:HDL for both treatments } \\
\qquad(p<0.05) \\
\downarrow \text { TC }(p=0.001) \text {, LDL }(p=0.001) \text { TC:LDL }(p=0.006) \\
\text { and LDL:HDL }(p=0.003) \text { for lupin relative to control. } \\
\text { No difference in HDL and TG. } \\
\text { No difference in PG for lupin } \\
(\downarrow \text { PG in control }(p=0.001)) \\
\text { No difference in PG, insulin, HOMA-IR or satiety } \\
\text { perception between treatments. } \\
\text { No difference in BW for either treatment }\end{array}$ \\
\hline $\begin{array}{l}\text { Johnson et al., } \\
2006^{*}[21]\end{array}$ & $\begin{array}{l}\text { Paper refers to the Hall } \\
2005 \text { study above }\end{array}$ & $\begin{array}{c}n=38 \\
\text { healthy men. } \\
\text { Mean age } 41.0 \pm 1.9 \text { years. } \\
\text { Mean BMI } 26.7 \pm 0.5 \mathrm{~kg} / \mathrm{m}^{2}\end{array}$ & As above & As above & As above & $\begin{array}{l}\text { Frequency and ease of } \\
\text { bowel motion, flatulence } \\
\text { level, Bristol Stool Form, } \\
\text { frequency (events), output, } \\
\text { transit time, pH, faecal } \\
\text { moisture content } \\
\text { SCFA (total, acetate, } \\
\text { propionate, isobutyrate, } \\
\text { butyrate, isovalerate, } \\
\text { valerate) }\end{array}$ & $\begin{array}{c}\uparrow \text { Frequency }(p=0.047), \\
\uparrow \text { faecal output }(p=0.020), \\
\downarrow \text { transit time }(p=0.012), \\
\uparrow \text { perception of flatulence level }(p<0.001), \\
\downarrow \text { faecal } \mathrm{pH}(p<0.001), \\
\uparrow \text { faecal moisture content }(p=0.027), \\
\uparrow \text { total SCFA concentration }(p=0.001) \text { and } \\
\uparrow \text { daily output }(p<0.001), \\
\uparrow \text { acetate concentration }(p<0.001) \text { and } \\
\uparrow \text { daily output }(p<0.001) \\
\uparrow \text { butyrate concentration }(p=0.006) \text { and output } \\
(p=0.002) \\
\uparrow \text { valerate output }(p=0.030) \text { with no difference in } \\
\text { concentration. } \\
\text { No difference in proprionate, isobutyrate or } \\
\text { isovalerate. }\end{array}$ \\
\hline
\end{tabular}


Table 4. Cont.

\begin{tabular}{|c|c|c|c|c|c|c|c|}
\hline Reference & Study Type & Subject $(n)$ and Characteristics & Intervention & Control/Comparator & $\begin{array}{l}\text { Energy } \\
\text { Balance }\end{array}$ & Main Health Markers & Main Outcomes \\
\hline $\begin{array}{l}\text { Fechner et al., } \\
2013 \text { [19] }\end{array}$ & $\begin{array}{l}\text { RCT double blind } \\
\text { cross-over study } \\
4 \text { periods of } 2 \text { weeks } \\
\text { each: run-in, } \\
2 \text { treatments and } \\
\text { washout }\end{array}$ & $\begin{array}{c}n=76 \\
\text { healthy men }(n=21) \text { and } \\
\text { women }(n=55) \text {. } \\
\text { Mean age } 24.4 \pm 3.2 \text { years. } \\
\text { Mean BMI } 21.7 \pm 2.4 \mathrm{~kg} / \mathrm{m}^{2}\end{array}$ & $\begin{array}{c}\text { Blue lupin kernel fibre and } \\
\text { white lupin kernel fibre. } \\
\text { Total dietary fibre per } \\
\text { treatment } 25 \mathrm{~g} / \mathrm{d} \text { in } \\
\text { beverages }\end{array}$ & $\begin{array}{l}\text { Citrus fibre as active } \\
\text { comparator for } 2 \text { lupin and } \\
1 \text { soya fibre treatments }\end{array}$ & Isocaloric & $\begin{array}{l}\text { TC, HDL, LDL, TG, faecal } \\
\text { pH, transit time, Bristol } \\
\text { Stool Form, faecal SCFAs } \\
\text { and bile acids }\end{array}$ & 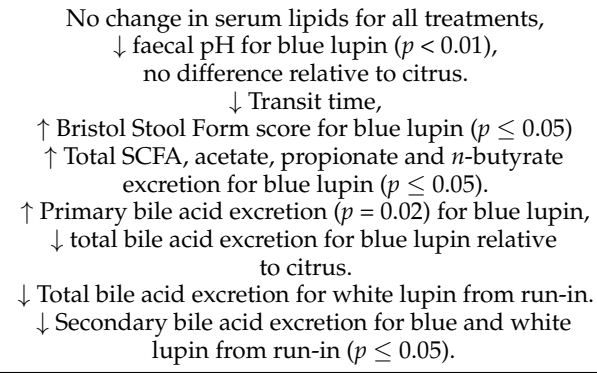 \\
\hline $\begin{array}{l}\text { Fechner et al., } \\
2014 \text { [29] }\end{array}$ & $\begin{array}{l}\text { RCT double blind } \\
\text { cross-over study } \\
3 \text { intervention periods } \\
\text { of } 4 \text { weeks each, run-in } \\
\text { and } 2 \text { washout periods } \\
\text { of } 2 \text { weeks each }\end{array}$ & $\begin{array}{c}n=52 \\
\text { moderately } \\
\text { hypercholesterol-aemic } \\
(\mathrm{TC}>5.2 \mathrm{mmol} / \mathrm{L}) \text { men }(n=20) \\
\text { and women }(n=32) . \\
\text { Mean age: } 46.9 \pm 3.2 \text { years. } \\
\text { Mean BMI: } 26.5 \pm 5.9 \mathrm{~kg} / \mathrm{m}^{2}\end{array}$ & $\begin{array}{l}\text { Blue lupin kernel fibre } \\
\qquad 25 \mathrm{~g} / \mathrm{d}\end{array}$ & $\begin{array}{l}\text { Citrus fibre } 25 \mathrm{~g} / \mathrm{d} \text { as } \\
\text { active comparator; control } \\
\text { diet (CD) with no } \\
\text { added fibre }\end{array}$ & Isocaloric & $\begin{array}{l}\text { General excretion markers, } \\
\text { faecal concentration or } \\
\text { excretion of neutral sterols, } \\
\text { bile acids and SCFAs. } \\
\text { BW, body composition, BP, } \\
\text { TC, HDL, LDL, TG } \\
\text { LDL:HDL hs-CRP, } \\
\text { satiety score }\end{array}$ & $\begin{array}{c}\downarrow \text { Faecal } \mathrm{pH} \text { from baseline }(p \leq 0.01) \text { and against CD } \\
\qquad p \leq 0.001), \\
\downarrow \text { transit time against } \mathrm{CD}(p \leq 0.05), \\
\text { no difference in neutral sterols. } \\
\uparrow \text { Primary bile acids from baseline }(p \leq 0.05), \\
\text { no difference in total or secondary bile acids. } \\
\uparrow \text { Formation of total SCFA from baseline }(p \leq 0.001) \\
\text { and against CD }(p \leq 0.01), \\
\uparrow \text { acetate from baseline and against CD }(p \leq 0.001), \\
\uparrow \text { propionate from baseline }(p \leq 0.001) \text { and against } \\
\text { control }(p \leq 0.05), \\
\begin{array}{c}\uparrow \text { butyrate from baseline }(p \leq 0.01) \text { and against control } \\
(p \leq 0.05) \text {. }\end{array} \\
\downarrow \text { BW, BMI, and WC from baseline }(p \leq 0.001) \text { and } \\
\text { against control }(p \leq 0.01) \text {. } \\
\downarrow \text { TC (9\%), LDL (12\%) and TG }(10 \%) \text { for lupin } \\
\text { compared with citrus }(p \leq 0.02), \\
\downarrow \text { hs-CRP }(p=0.02) \text {, SBP }(p=0.01) \text { for lupin compared } \\
\text { to baseline. } \\
\uparrow \text { Perception of satiety }(p \leq 0.001)\end{array}$ \\
\hline
\end{tabular}

* Part of one study; Abbreviations: Body mass index (BMI); Blood pressure (BP)); Body weight (BW); High density lipoprotein cholesterol (HDL); Homeostasis model assessment of insulin resistance (HOMA-IR); High-sensitivity C-reactive protein (hs-CRP); Low density lipoprotein cholesterol (LDL); Plasma glucose (PG); Short-chain fatty acid (SCFA); Triglycerides (TG); Total cholesterol (TC). 


\subsection{Risk of Bias}

Each study was assessed according to the criteria outlined in the revised Cochrane RoB2 tool for RCTs. All parallel studies had a low risk of bias. Most cross-over studies had a low risk of bias (Figure 2); exceptions were one study with some concern for risk of bias in Domain1: Randomisation process, and in Domain 5: Selection of the reported result [25]. One other cross-over study had some concern in Domain 1 only [24].

Domain 1: Randomisation process

Domain S: Bias arising from period and carryover effects

Domain 2: Deviations from intended interventions

Domain 3: Missing outcome data

Domain 4: Measurement of the outcome

Domain 5: Selection of the reported result

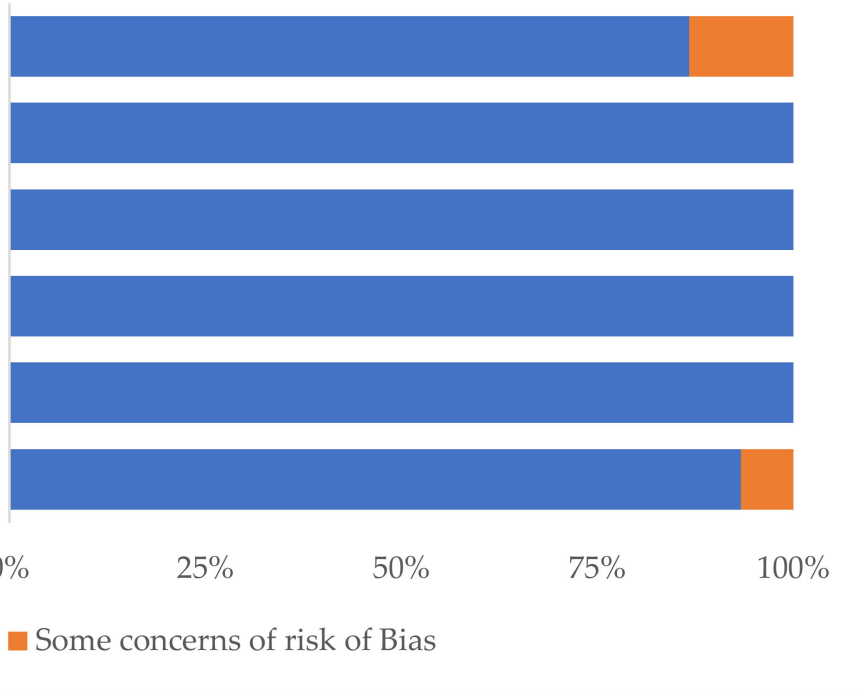

Low risk of Bias

- Some concerns of risk of Bias

Figure 2. Within-study risk of bias assessment using the revised Cochrane risk-of-bias tool (RoB2) in 15 randomised $(n=11)$ and non-randomised $(n=2)$ controlled cross-over trials examining health outcomes of lupin consumption.

\subsection{Range of Investigated Health Measurements and Their Outcomes}

The five most investigated variables or groups of variables across all studies and the direction of lupin consumption effect are shown in Figure 3. These were:

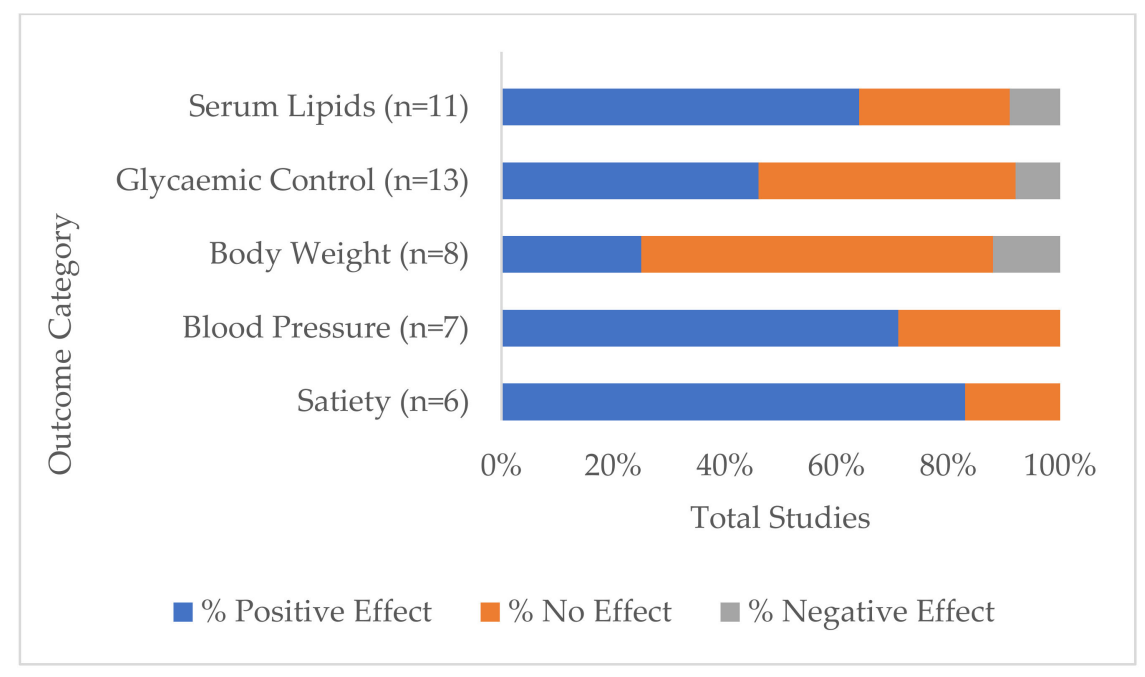

Figure 3. Percentage of total studies that reported differences between baseline and/or comparators $(p<0.05)$ by the five most investigated groups of health markers: serum lipids, glycaemic control, body weight, blood pressure and satiety, that had positive (desirable), negative (detrimental) or no effect on health outcomes. 
Serum lipids, which included any one or more of total cholesterol, LDL and HDL cholesterol, LDL:HDL ratio and triglycerides. Eleven studies measured serum lipids refs. $[19,22,23,25,27-31,34,35]$, of which $64 \%$ had one or more positive outcomes (i.e., showed statistically significant within-study difference(s) from baseline and/or control in a direction considered optimal for good health, depending on the health marker tested). Three studies did not report differences in serum lipids [19,23,34], while one study reported reduced HDL cholesterol with other lipids unchanged [35].

I. Glycaemic control included measurements of post-prandial and long-term glucose and insulin levels, HOMA-IR and HbA1c. These were measured in 13 studies refs. $[15-18,22-28,34,35]$ with positive effects indicated in $46 \%$ of these studies refs. [15-17,24,26,35].

II. Body weight measurements were monitored in 8 studies [22,23,25,28-30,34,35], of which $25 \%$ recorded unintended weight loss [25,29], while $12.5 \%$ (one study) recorded increased body weight [30].

III. Blood pressure was measured in 7 studies [23,25,29-31,33,35], two of which did not find significant differences $[23,31]$ while the remaining five $(71 \%)$ recorded reductions.

IV. Perceptions of satiety were monitored in 6 studies [16-18,22,24,29]. A significantly higher satiety rating score was given for lupin in $83 \%$ of studies, while one study saw no difference to control [22].

Variables measured in five or fewer studies included those relating to inflammation, such as hs-CRP [28-31,35], IL-6 and sICAM-1 [28], oxidative stress [32], and those relating to food intake [16-18,29], appetite, digestion and metabolism, such as concentrations of serum ghrelin [16], leptin [34], adiponectin [28,34], bowel function [19,21,24,29], faecal SCFA concentrations $[19,21,29]$, faecal bile acid concentrations [19,29] and faecal microbiome varieties and populations [20]. No studies found significant differences in inflammation and oxidative stress markers, nor in the gastric hormones and adiponectin. However, bowel function changes were seen in all but one of the studies [24]. All studies that measured faecal SCFA concentrations and the faecal microbiome composition study found significant changes.

\subsection{Range of Health Outcomes by Lupin Treatment Category \\ 3.5.1. Whole Lupin}

Seventy-five percent $(n=9)$ of all whole lupin studies $(n=12)$ reported a significant difference in at least one of the health markers under investigation, compared to baseline or to the control group. While most of the directions of change were desirable or positive outcomes for that variable, one study of overweight and obese participants reported decreased levels of HDL cholesterol, thereby reducing its potential heart-protective benefit, while other lipid markers did not change [35]. One study [25] reported increased serum glucose at 14 and 28 weeks and increased insulin levels at 28 weeks following a daily lupin snack in the first phase and two lupin snacks per day in the subsequent phase. Key health outcomes for whole lupin are shown in Figure 4a. All whole lupin studies $(n=4)$ that measured perceptions of satiety reported desirable changes [16-18,24], as well as $75 \%$ $(n=3)$ of studies that monitored blood pressure $(n=4)[25,33,35]$ and $67 \%(n=6)$ of studies that measured glycaemic control $(n=10)[15-17,24,26,35]$. Serum lipids moved to healthier levels in $25 \%(n=1)$ of studies $(n=4)$ [25], as well as reporting decreased body weight measurements [25]. 


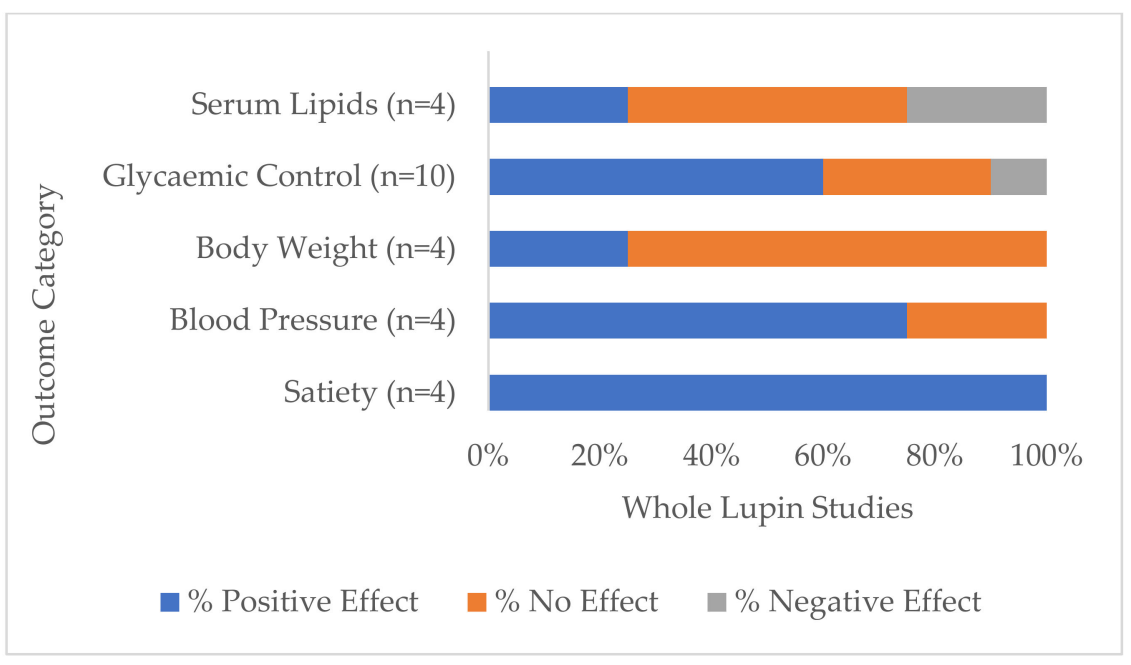

(a)

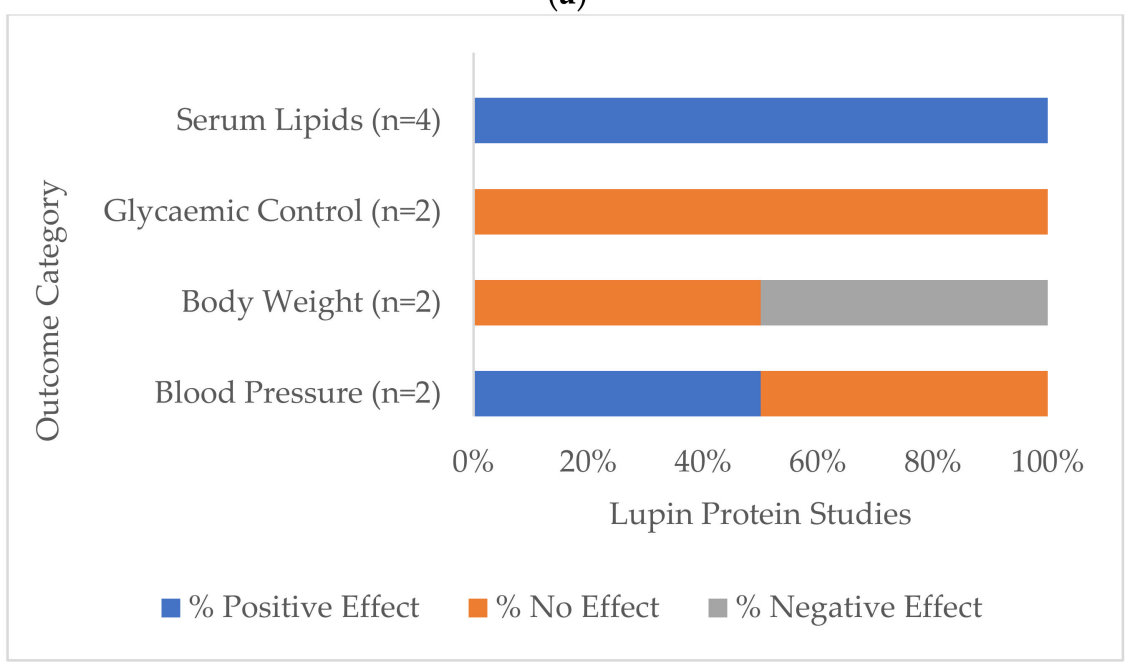

(b)

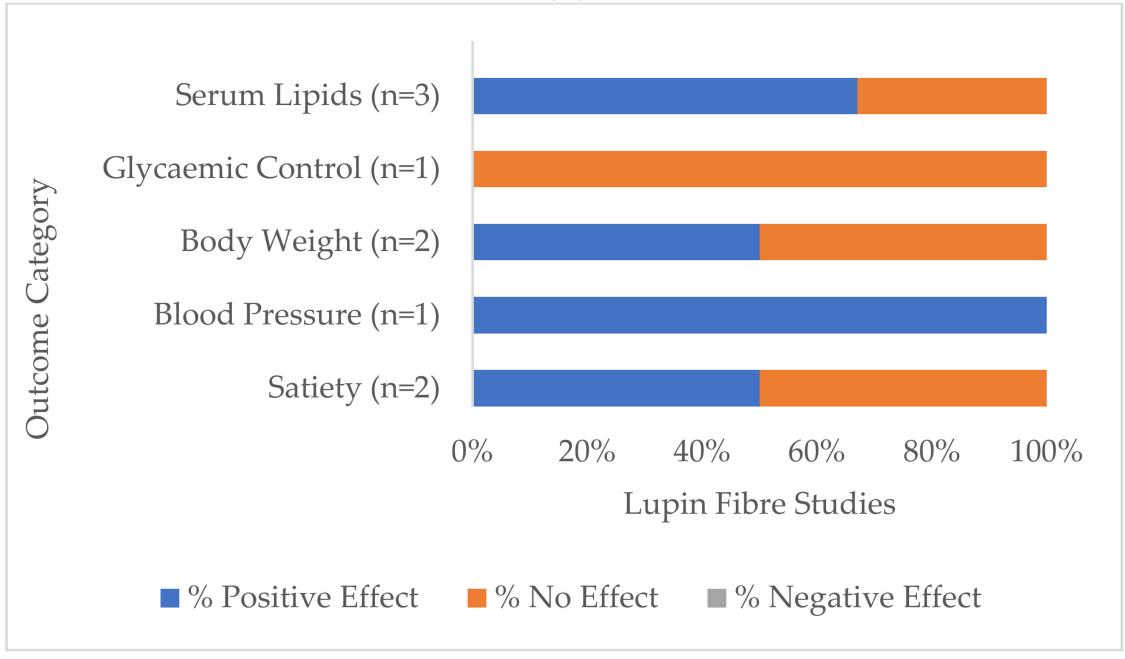

(c)

Figure 4. Percentage of total studies categorised by (a) whole lupin, (b) lupin protein and (c) lupin fibre treatment that reported positive (potentially beneficial), negative (potentially detrimental) and no significant differences between baseline and/or comparators $(p<0.05)$ in the five most investigated groups of health markers: serum lipids, glycaemic control, body weight, blood pressure and satiety. 


\subsubsection{Lupin Protein}

All lupin protein studies $(n=4)$ reported significant differences in at least one of the targeted health markers. All studies that measured serum lipids [27,28,30,31] and one study [30] of a total of two that measured blood pressure [30,31] reported significantly reduced levels (Figure $4 b$ ).

\subsubsection{Lupin Fibre}

All lupin fibre studies $(n=5)$ reported significant differences in at least one of the measured health markers. Two out of the three lupin fibre studies that measured serum lipids reported significant differences and positive health outcomes [22,29], as well as the one study that measured blood pressure [29] (Figure 4c). All three lupin fibre studies that monitored bowel function reported positive changes [19,21,29].

\section{Discussion}

This systematic review of the evidence for health outcomes from lupin consumption observed a range of results across many biological and anthropometric health markers that variously resulted in no effects, positive effects and negative effects in terms of optimum health. In the 21 studies that met the selection criteria, the strongest evidence related to a lowering effect on total cholesterol, LDL cholesterol and LDL cholesterol:HDL cholesterol ratio; reduction in systolic blood pressure, increased satiety and improvement in postprandial and glycaemic control. This supports the notion that lupin is equally and possibly more effective among all legumes in protecting long-term health. After categorising studies by form of lupin utilised in each intervention, this review noted potential relationships between lupins in their whole form and increased satiety perception, decreased blood pressure and improved glycaemic control, whereas lupin protein and lupin fibre demonstrated strongest positive results for blood pressure and serum lipids (though from a small study base).

These results correspond with the Kouris-Blazos et al. [8] review which concluded that sweet lupins may favourably effect blood pressure, blood lipids, insulin sensitivity and the gut microbiome. The Prusinski review [11] of white lupin concurred, stating that people who experience health conditions such as diabetes, hypertension, obesity, cardiovascular disease, hyperlipidaemia and colorectal cancer may benefit from the incorporation of this legume in the diet. However, research for the review centred on physiological properties of white lupin rather than on evidence for actual health outcomes. A 2017 review and meta-analyses investigating relationships between mortality and the intake of various food groups [36] found an inverse association between all-cause mortality and increased consumption of legumes, with no further dose response after $150 \mathrm{~g} /$ per day.

\subsection{Whole Lupin}

Nutrients in foods are metabolised in the human body according to the food matrix [37]. Categorisation of studies in this review by type of lupin administration, i.e., whole lupin, its protein and its fibre component, revealed greater health benefits were observed for the consumption of the whole food. Improved health outcomes were consistent for blood pressure [25,33,35], satiety [16-18,24] and glycaemic control markers [15-17,24,26,35] in whole lupin treatment studies, indicating benefits for reducing risk and managing symptoms of hypertension, cardiovascular disease, diabetes and obesity. Although it was noted that evidence for an increased satiety effect was present, evidence was less convincing for weight loss. None of the three whole lupin studies that measured body measurements detected a significant reduction in body weight $[23,34,35]$. Several reasons may account for this observation. Participants followed ad libitum diets that were not intended for weight loss, other lifestyle factors impacting weight, such as physical activity, were not monitored, and treatment duration may have been too short to demonstrate significant change. Hodgson et al. [34] proposed that if the observed trend in weight loss after four months was extended, a significant reduction of $2 \mathrm{~kg}$ could be expected within two years. 
The study further proposed that while ad libitum diets that are high in protein and dietary fibre may result in loss of body weight, the amount of protein and fibre in whole lupin may be a factor. In addition, the mostly insoluble fibre present in lupin may not be as effective as isolated soluble fibre used in many dietary fibre weight loss studies. Nevertheless, the broad health benefits proposed by whole lupin consumption suggest a synergistic interplay of macro- and micronutrient components within the whole food matrix and their influence on multiple biological functions [37], leading to improved long-term health outcomes.

\subsection{Lupin Protein and Fibre Components}

Protein and dietary fibre components of lupin individually demonstrated consistent evidence for lipid lowering effects [22,27-31]. These benefits were observed in hypercholesterolaemic participants, as well as in one of two studies that recruited healthy participants, yet whose average baseline total cholesterol was above $5 \mathrm{mmol} / \mathrm{L}$ [22]. Given that lipid levels did not change significantly in the lupin component study based on healthy men and women with average baseline total cholesterol below $5 \mathrm{mmol} / \mathrm{L}$ [19], this suggests lupin protein and fibre had moderating effects above this level. Evidence for blood pressure reduction from lupin component interventions with that of whole lupin does not conclusively favour its protein or fibre alone due to the paucity of studies. Serum lipids were measured by a similar number of studies across all three categories of lupin treatment. While results were variable, mostly positive effects were observed in diets that contained isolated lupin protein and lupin fibre. Substantially higher quantities of protein and fibre were provided in component trial protocols, compared with the amounts obtainable from the whole food in whole lupin studies. This may have contributed to the more consistently positive lipid outcomes in these lupin component studies. While the evidence for any health benefits from isolated lupin component consumption cannot be confirmed from so few studies, the addition of lupin protein to foods and beverages for maximising protein intake may be a useful alternative to soy and whey protein, particularly for consumers avoiding phytoestrogens and animal proteins. Similarly, lupin fibre is a gluten-free alternative for individuals with coeliac disease.

\subsection{Dose Response}

Indication of a dose response relationship between lupin and health outcomes was not identified due to multiple forms of lupin delivery and study methods among studies. However, one study [25] designed a dose response protocol comprising a doubled intake of whole Lupinus mutabilis during one intervention phase from $10 \mathrm{~g}$ to $20 \mathrm{~g}$ per day. While there was no change in glycaemic response markers between the two doses, blood pressure reduction was greatest after the increased dose phase.

\subsection{Healthy vs. Unhealthy Participants Health Outcomes}

Substantial evidence for health marker differences between healthy participants and those with type 2 diabetes, hypercholesterolaemia or who were overweight or obese, was unable to be determined because of the heterogeneity among studies. Having undertaken a sub-group analysis, however, one lupin fibre study [22] on generally healthy subjects found no significant effect on serum lipids among normocholesterolaemic participants (baseline total cholesterol $<5.5 \mathrm{mmol} / \mathrm{L}$ ), while LDL cholesterol was significantly lowered among participants identified by study authors as 'mild to clinically hypercholesterolaemic' (baseline total cholesterol $>5.5 \mathrm{mmol} / \mathrm{L}$ ). The investigation of whole Lupinus mutabilis consumption in type 2 diabetic subjects under conventional non-insulin medication found significantly reduced glycosylated haemoglobin $(\mathrm{HbA1c})$ outcomes among a sub-group with less severe disease ( $\mathrm{HbA1c}$ maintained at $\leq 8 \%$ ), while no $\mathrm{HbA1c}$ effect was found in the remaining group that maintained $\mathrm{HbA1C} \geq 8 \%$ and $<10 \%$ [25]. Though changes in biomarkers may not be demonstrated consistently in healthy subjects, it can be supposed that lupin consumption offers protective benefits in hypercholesterolaemia and in wellcontrolled hyperglycaemia, if not in disease of greater severity. 


\subsection{Progression of Lupin and Health Outcomes Knowledge}

Reflection on 16 years of lupin and health research revealed a progression from a focus on principal biomarkers for chronic disease, to a broader scope that encompassed other related health markers and possible biological mechanisms of effect. Analysis of faecal SCFA composition after lupin fibre consumption proposed that increased concentration and output of acetate and butyrate may have a protective effect on colorectal cancer risk [21]. A study on lupin fibre that observed a reduction in serum lipids in hypercholesterolaemic individuals proposed that increased bile acid excretion was not the result of bile acids binding to fibre, but a lower environmental $\mathrm{pH}$ from the fermentation of lupin fibre in the gut and the subsequent release of SCFAs [29]. Since the single study that focussed on lupin fibre and faecal gut bacteria was in 2006 [20], current interest and greater understanding of the gut microbiome warrants further investigation.

\subsection{Strengths and Limitations}

This systematic literature review was undertaken with the acknowledgement of several strengths and limitations. A major strength was the inclusion of high quality RCTs and non-randomised controlled studies that disclosed valid contextual reasons for nonrandomisation. Implementation of the revised Cochrane risk-of-bias tool facilitated recognition and acknowledgement of any limitations within studies. Limitations within this review relate to potential publication bias as only research published in English language journals was targeted. Relatively few studies met the selection criteria and the participant base was limited. Furthermore, the objectives, methods and analyses of the various studies lacked homogeneity, thus precluding a meta-analysis to be performed.

\subsection{Future Directions}

The subject of lupin consumption and health outcomes is a relatively new area of investigation, therefore more research is required to expand the evidence base. This should comprise multiple studies with similar aims, designs and protocols based on adequately sized population groups. Studies should identify the species of lupin and test all lupin forms in quantities that could feasibly be included in a normal diet, preferably in a dose response manner. This would allow for a more accurate assessment of the evidence overall for health benefits and optimum intake. In terms of health outcome measures, those for blood lipids, blood pressure and glycaemic control would be the most useful in identifying the unique nutritional and physiological properties of lupin. Furthermore, studies that involve concurrent investigations on healthy populations and those with different degrees of disease severity will inform whether lupin consumption may be more useful as a riskreduction strategy or in chronic disease minimisation.

\section{Conclusions}

This is the first systematic review to our knowledge to investigate the range of health outcomes and lupin consumption according to its mode of delivery, either as a whole food or the protein or fibre component. This review found divergent results in the effects of lupin consumption on many health marker outcomes, though greatest indications of benefit were apparent in improved satiety and reductions in blood pressure, and to a lesser extent in reductions in serum lipids and improved glycaemic control. More often, evidence was based on the whole lupin providing a broader range of health benefits than was observed in the smaller number of component studies. While the evidence for lupin's health benefits is promising, more substantial research would be required before health claims could be made. Nevertheless, its unique nutritional and physiological properties, particularly as a whole food, make it an ideal legume to include in a healthy diet. 
Supplementary Materials: The following are available online at https:/ / www.mdpi.com/article /10.3390/nu14020327/s1, Table S1: PICO (Population, Intervention, Comparator/Control, Outcome) framework to define the search strategy for the question: 'Is there an effect of human lupin consumption on health outcomes?', File S2: Search terms (MEDLINE).

Author Contributions: Conceptualization, L.B., S.G. and A.R.; methodology, L.B. and S.G.; formal analysis, L.B.; writing-original draft preparation, L.B.; writing-review and editing, L.B., S.G. and A.R.; supervision, S.G. and A.R. All authors have read and agreed to the published version of the manuscript.

Funding: This research received no external funding however was supported by the Grains \& Legumes Nutrition Council, a not-for-profit charity organisation.

Conflicts of Interest: S.G. was employed by the Grains \& Legumes Nutrition Council, a not-for-profit charity organization, during the period when the search was conducted. L.B. and A.R. declare no conflict of interest.

\section{References}

1. Grains \& Legumes Nutrition Council (G.L.N.C). Lupins. Available online: https://www.glnc.org.au/legumes/types-of-legumes /lupins/ (accessed on 23 August 2021).

2. NSW Government Industry \& Investment. Lupin Growth \& Development. Available online: https://www.dpi.nsw.gov.au/_dat a/assets / pdf_file/0006/516183/Procrop-lupin-growth-and-development.pdf (accessed on 23 August 2021).

3. Foyer, C.H.; Lam, H.-M.; Nguyen, H.T.; Siddique, K.H.M.; Varshney, R.K.; Colmer, T.D.; Cowling, W.; Bramley, H.; Mori, T.A.; Hodgson, J.M.; et al. Neglecting legumes has compromised human health and sustainable food production. Nat. Plants 2016, 2, 16112. [CrossRef] [PubMed]

4. Food Standards Australia New Zealand (FSANZ). Australian Sweet Lupin. Available online: https://www.foodstandards.gov.au / science/monitoringnutrients/afcd/Documents/Industry\%20Report\%20-\%20Australian\%20Sweet\%20Lupin.pdf (accessed on 23 August 2021).

5. Grains \& Legumes Nutrition Council (G.L.N.C). Consumption of Attitudes Study Results: 2017. Available online: https: //www.glnc.org.au/wp-content/uploads/2018/09/GLNC-Consumption-Study-Summary-5.pdf (accessed on 23 August 2021).

6. Figueira, N.; Curtain, F.; Beck, E.; Grafenauer, S. Consumer understanding and culinary use of legumes in Australia. Nutrients 2019, 11, 1575. [CrossRef] [PubMed]

7. National Health and Medical Research Council (NHMRC). A Modelling System to Inform the Revision of the Australian Guide to Healthy Eating; Department of Health and Ageing: Canberra, Australia, 2011; Volume 66.

8. Kouris-Blazos, A.; Belski, R. Health benefits of legumes and pulses with a focus on Australian sweet lupins. Asia Pac. J. Clin. Nutr. 2016, 25, 1-17. [PubMed]

9. Food Standards Australia New Zealand (FSANZ). Allergen Labelling. Available online: https://www.foodstandards.gov.au/con sumer/labelling/Pages/Allergen-labelling.aspx (accessed on 12 November 2021).

10. Allergy and Anaphylaxis Australia. Lupin. Available online: https://allergyfacts.org.au/allergy-anaphylaxis/food-allergens/1 upin (accessed on 25 November 2021).

11. Janusz, P. White lupin (Lupinus albus L.) -Nutritional and health values in human nutrition-A review. Czech J. Food Sci. 2017, 35, 95-105. [CrossRef]

12. Bielefeld, D.; Grafenauer, S.; Rangan, A. The effects of legume consumption on markers of glycaemic control in individuals with and without diabetes mellitus: A systematic literature review of randomised controlled trials. Nutrients 2020, 12, 2123. [CrossRef]

13. Moher, D.; Liberati, A.; Tetzlaff, J.; Altman, D.G. Preferred reporting items for systematic reviews and meta-analyses: The PRISMA statement. Ann. Intern. Med. 2009, 151, 264-269. [CrossRef]

14. Sterne, J.A.; Savović, J.; Page, M.J.; Elbers, R.G.; Blencowe, N.S.; Boutron, I.; Cates, C.J.; Heng, H.-Y.; Corbett, M.S.; Eldridge, S.M. RoB 2: A revised tool for assessing risk of bias in randomised trials. BMJ 2019, 366, 14898. [CrossRef]

15. Schopen, K.; Ewald, A.C.; Johannes, B.W.; Bloch, W.; Rittweger, J.; Frings-Meuthen, P. Short-term effects of lupin vs. Whey supplementation on glucose and insulin responses to a standardized meal in a randomized cross-over trial. Front. Physiol. 2017, 8, 198. [CrossRef]

16. Lee, Y.P.; Mori, T.A.; Sipsas, S.; Barden, A.; Puddey, I.B.; Burke, V.; Hall, R.S.; Hodgson, J.M. Lupin-enriched bread increases satiety and reduces energy intake acutely. Am. J. Clin. Nutr. 2006, 84, 975-980. [CrossRef]

17. Keogh, J.; Atkinson, F.; Eisenhauer, B.; Inamdar, A.; Brand-Miller, J. Food intake, postprandial glucose, insulin and subjective satiety responses to three different bread-based test meals. Appetite 2011, 57, 707-710. [CrossRef]

18. Hall, R.S.; Thomas, S.J.; Johnson, S.K. Australian sweet lupin flour addition reduces the glycaemic index of a white bread breakfast without affecting palatability in healthy human volunteers. Asia Pac. J. Clin. Nutr. 2005, 14, 91-97.

19. Fechner, A.; Fenske, K.; Jahreis, G. Effects of legume kernel fibres and citrus fibre on putative risk factors for colorectal cancer: A randomised, double-blind, crossover human intervention trial. Nutr. J. 2013, 12, 101. [CrossRef] 
20. Smith, S.C.; Choy, R.; Johnson, S.K.; Hall, R.S.; Wildeboer-Veloo, A.C.; Welling, G.W. Lupin kernel fiber consumption modifies fecal microbiota in healthy men as determined by rRNA gene fluorescent in situ hybridization. Eur. J. Nutr. 2006, 45, 335-341. [CrossRef]

21. Johnson, S.K.; Chua, V.; Hall, R.S.; Baxter, A.L. Lupin kernel fibre foods improve bowel function and beneficially modify some putative faecal risk factors for colon cancer in men. Br. J. Nutr. 2006, 95, 372-378. [CrossRef]

22. Hall, R.S.; Johnson, S.K.; Baxter, A.L.; Ball, M.J. Lupin kernel fibre-enriched foods beneficially modify serum lipids in men. Eur. J. Clin. Nutr. 2005, 59, 325-333. [CrossRef]

23. Ward, N.C.; Mori, T.A.; Beilin, L.J.; Johnson, S.; Williams, C.; Gan, S.K.; Puddey, I.B.; Woodman, R.; Phillips, M.; Connolly, E.; et al. The effect of regular consumption of lupin-containing foods on glycaemic control and blood pressure in people with type 2 diabetes mellitus. Food Funct. 2020, 11, 741-747. [CrossRef]

24. Skalkos, S.; Moschonis, G.; Thomas, C.J.; McMillan, J.; Kouris-Blazos, A. Effect of lupin-enriched biscuits as substitute mid-meal snacks on post-prandial interstitial glucose excursions in post-surgical hospital patients with type 2 diabetes. Nutrients $\mathbf{2 0 2 0}$ 12, 1239. [CrossRef]

25. Fornasini Salvador, M.V.; Abril-Ulloa, S.V.; Beltran Carreno, J.P.; Villacres, E.; Cuadrado-Merino, L.; Robalino, F.; Sanchez, R.; Ricaurte Ortiz, P.S.; Munoz, E.B.; Benitez Loza, N.B.; et al. Efficacy of a Lupinus mutabilis Sweet snack as complement to conventional type 2 diabetes mellitus treatment. Nutr. Hosp. 2019, 36, 905-911. [CrossRef]

26. Dove, E.R.; Mori, T.A.; Chew, G.T.; Barden, A.E.; Woodman, R.J.; Puddey, I.B.; Sipsas, S.; Hodgson, J.M. Lupin and soya reduce glycaemia acutely in type 2 diabetes. Br. J. Nutr. 2011, 106, 1045-1051. [CrossRef]

27. Weisse, K.; Brandsch, C.; Zernsdorf, B.; Nkengfack Nembongwe, G.S.; Hofmann, K.; Eder, K.; Stangl, G.I. Lupin protein compared to casein lowers the LDL cholesterol:HDL cholesterol-ratio of hypercholesterolemic adults. Eur. J. Nutr. 2010, 49, 65-71. [CrossRef]

28. Sirtori, C.R.; Triolo, M.; Bosisio, R.; Bondioli, A.; Calabresi, L.; De Vergori, V.; Gomaraschi, M.; Mombelli, G.; Pazzucconi, F.; Zacherl, C.; et al. Hypocholesterolaemic effects of lupin protein and pea protein/fibre combinations in moderately hypercholesterolaemic individuals. Br. J. Nutr. 2012, 107, 1176-1183. [CrossRef]

29. Fechner, A.; Kiehntopf, M.; Jahreis, G. The formation of short-chain fatty acids is positively associated with the blood lipidlowering effect of lupin kernel fiber in moderately hypercholesterolemic adults. J. Nutr. 2014, 144, 599-607. [CrossRef]

30. Bahr, M.; Fechner, A.; Kramer, J.; Kiehntopf, M.; Jahreis, G. Lupin protein positively affects plasma LDL cholesterol and LDL:HDL cholesterol ratio in hypercholesterolemic adults after four weeks of supplementation: A randomized, controlled crossover study. Nutr. J. 2013, 12, 107. [CrossRef]

31. Bahr, M.; Fechner, A.; Kiehntopf, M.; Jahreis, G. Consuming a mixed diet enriched with lupin protein beneficially affects plasma lipids in hypercholesterolemic subjects: A randomized controlled trial. Clin. Nutr. 2015, 34, 7-14. [CrossRef] [PubMed]

32. Yang, X.; Croft, K.D.; Lee, Y.P.; Mori, T.A.; Puddey, I.B.; Sipsas, S.; Barden, A.; Swinny, E.; Hodgson, J.M. The effects of a lupin-enriched diet on oxidative stress and factors influencing vascular function in overweight subjects. Antioxid. Redox Signal. 2010, 13, 1517-1524. [CrossRef]

33. Lee, Y.P.; Mori, T.A.; Puddey, I.B.; Sipsas, S.; Ackland, T.R.; Beilin, L.J.; Hodgson, J.M. Effects of lupin kernel flour-enriched bread on blood pressure: A controlled intervention study. Am. J. Clin. Nutr. 2009, 89, 766-772. [CrossRef]

34. Hodgson, J.M.; Lee, Y.P.; Puddey, I.B.; Sipsas, S.; Ackland, T.R.; Beilin, L.J.; Belski, R.; Mori, T.A. Effects of increasing dietary protein and fibre intake with lupin on body weight and composition and blood lipids in overweight men and women. Int. J. Obes. 2010, 34, 1086-1094. [CrossRef]

35. Belski, R.; Mori, T.A.; Puddey, I.B.; Sipsas, S.; Woodman, R.J.; Ackland, T.R.; Beilin, L.J.; Dove, E.R.; Carlyon, N.B.; Jayaseena, V.; et al. Effects of lupin-enriched foods on body composition and cardiovascular disease risk factors: A 12-month randomized controlled weight loss trial. Int. J. Obes. 2011, 35, 810-819. [CrossRef] [PubMed]

36. Schwingshackl, L.; Hoffmann, G.; Iqbal, K.; Schwedhelm, C.; Boeing, H. Food groups and intermediate disease markers: A systematic review and network meta-analysis of randomized trials. Am. J. Clin. Nutr. 2018, 108, 576-586. [CrossRef] [PubMed]

37. Melse-Boonstra, A. Bioavailability of micronutrients from nutrient-dense whole foods: Zooming in on dairy, vegetables, and fruits. Front. Nutr. 2020, 7, 101. [CrossRef] 